\title{
Aus dem Vorwort
}

Die Anregung zu diesem Buch kam von einer Vorlesung, die ich 1967 in London während der Hauptversammlung der Association for Science Education hielt. Sie hatte sich zum Ziel gesetzt zu zeigen, welche experimentellen Methoden zur Aufklärung von Reaktionsabläufen herangezogen und wie ihre Ergebnisse so zusammengefügt werden können, da $B$ sich daraus die molekularen Ereignisse ablesen lassen. Einige Hörer der Vorlesung betonten, daß sie diese Art der Darstellung als anregend empfunden hatten, daß sie aber nicht wüßten, wo sie etwas Ähnliches nachlesen könnten. Es schien daher, daß ein einfach gehaltenes Buch über dieses Thema von Nutzen sein könnte: Dies ist der Versuch eines solchen Buches.

Die Anlage dieses Buches dürfte aus den Bezeichnungen der einzelnen Kapitel klar hervorgehen. Ich hoffe, eine nützliche und funktionsgerechte Gliederung des Stoffes gefunden zu haben. Mit den kinetischen Daten und ihrer Interpretation zu beginnen, scheint nicht nur logisch, sondern sogar notwendig, denn hier liegt die eigentliche Wurzel all unserer Vorstellungen über Reaktionsabläufe. Die folgenden Kapitel sind dann so angelegt, daß nach und nach immer kompliziertere Argumente und immer schwierigere Situationen behandelt werden. Das letzte Kapitel bringt dann einige weitere Beispiele, zu deren Erörterung es einer Fortentwicklung der zuvor besprochenen Methoden und Schlußweisen oder auch ihrer kombinierten Anwendung bedarf, damit schließlich noch nähere Einzelheiten über den Ablauf bestimmter Reaktionen angegeben werden können. Es sollte gesagt werden, daß dieses Buch bei alledem einfach geschrieben ist. Es wird nichts oder fast nichts für sein Verständnis vorausgesetzt, was über die elementarsten Kenntnisse der Organischen Chemie hinausginge. Allerdings wird, vor allem in den späteren Kapiteln, eine sorgfältige Mitarbeit des Lesers vorausgesetzt. 
